\title{
Aislamiento e identificación de microalgas clorofitas para la producción de lípidos como fuente de biodiesel
}

Isolation and identification of microalgae chlorophytes for the production of lipids as a source of biodiesel

\section{Luis Alberto Huayna D ${ }^{1}$., José Luis Romero B. ${ }^{1}$, William Guzmán S. ${ }^{1}$, Adelfio Bacilio A. ${ }^{1}$,} Ricardo Alor $\mathrm{S}^{2}$., Jean Piere Quiliche D. ${ }^{1}$

\section{RESUMEN}

Objetivo: Determinar las especies más representativas de microalgas clorofitas aisladas e identificadas de la bahía de Huacho con potencial aplicación para la producción de biodiesel. Métodos: Fue una investigación explicativa experimental. La población estuvo conformada por flora marina o sea el fitoplancton donde se encuentran las microalgas clorofitas. La muestra fue de tipo no estadístico tomada en tres puntos de muestreo. Se evaluaron cuatro métodos de aislamiento y purificación: rayado en placas de agar, diluciones sucesivas y subcultivos repetidos, pipeteo capilar y cultivo en fotobiorreactores en medios líquidos. Para la identificación se realizaron preparados húmedos de las colonias de microalgas clorofitas aisladas utilizando el microscopio de campo claro para la observación. Resultados: Se obtuvieron cuatro cepas unialgales de clorofitas: Nannochloropsis sp., Chlorella sp., Navicula sp. y Spirulina sp.; correspondiendo al Nannochloropsis sp. quien presentó mayor densidad poblacional en el medio de cultivo sólido F/2 Guillard-Ryther. Conclusión: Las muestras de fitoplancton marino presentaron poca cantidad de microalgas clorofitas (algas verdes) confirmando lo mencionado por algunos autores.

Palabras clave: Fitoplancton, microalgas clorofitas, cultivo heterotrófico, fotobiorreactor.

\section{ABSTRACT}

Objetive: Determine the most representative species of chlorophytes microalgae isolated and identified of the Huacho bay with potential application in the production of biodiesel. Methods: It was an experimental explanatory research. The population consisted of marine plant or phytoplankton is where the chlorophytes microalgae. The statistical sample was not taken in kind three sampling points. Four methods of isolation and purification were evaluated: agar plates striped, successive dilutions and repeated subcultures, capillary pipetting and liquid culture media photobioreactors. For identification were performed wet preparations algae Chlorophyta colonies isolated using the light microscope for observation. Results: four unialgal chlorophytes strains were isolated: Nannochloropsis sp., Chlorella sp., Navicula sp. and Spirulina sp.; Nannochloropsis sp. corresponding who presented the highest population density in the solid medium F/2GuillardRyther. Conclusion: marine phytoplankton samples showed little amount of chlorophytes microalgae (green algae) confirming mentioned by some authors.

Keywords: Phytoplankton, microalgae chlorophytes, heterotrophic culture, photobioreactor.

\footnotetext{
${ }^{1}$ Facultad de Ciencias. Universidad Nacional José Faustino Sánchez Carrión. Huacho, Perú.

${ }^{2}$ Facultad de Ingeniería Pesquera. Universidad Nacional José Faustino Sánchez Carrión. Huacho, Perú.
} 


\section{INTRODUCCIÓN}

Debido al agotamiento de las reservas de petróleo y a las consecuencias ambientales del uso excesivo de los combustibles fósiles, la producción de combustibles alternativos posee gran atención (Ma y Hanna, 1999; Chisti, 2007; Song, Fu y Shi, 2008; Meng et al., 2008; Kalia y Purohit, 2008). Actualmente las fuentes existentes de energía son la energía termonuclear, solar, eólica, geotérmica (Veziroglu y Barbir, 1992). De entre los reemplazos a los combustibles fósiles, el Biodiesel es uno de los más importantes (Ma y Hanna, 1999), ya que puede ser producido a partir de aceites vegetales, grasa de cocina reciclada, aceites de desecho, grasa animal y de lípidos provenientes de microalgas (Van Gerpen 2005 y Song et al., 2008).

Algunos autores han mencionado que las diatomeas (Bacillariophyta), los dinoflagelados (Dinoflagellata), las Haptophyta y las algas verdes (Chlorophyta) podrían contar aproximadamente por el 40 , 40,10 y $6 \%$ de las especies de eucariotas presentes en el fitoplancton marino actual, mientras que la suma de otros grupos contribuiría un 2\% (Simon et al., 2009). Las algas microscópicas han merecido considerable atención por su potencialidad como fuente de biodiesel, por la posibilidad de suplir de manera parcial la escasez de suministro de petróleo y asimismo, se presentan como una alternativa a los precios elevados de las materias primas tradicionales, para la obtención de los biocombustibles como son la soja, caña de azúcar, maíz, palma aceitera, colza, grasas animales, residuos de grasas, entre los más representativos (Knothe Dunn y Bagby 1997; Lee, 1997 y Garibay et al., 2009).

Dependiendo de las especies, las microalgas producen diferente tipos de lípidos, hidrocarburos y otros aceites complejos (Álvarez y Zarco, 1989; Guschina y Harwood, 2006), de los cuales no todos son adecuados para producir biodiesel. Arias, Martínez y Cañizares (2013) e Hirata et al. (2001) señalaron que entre las microalgas de potencial importancia para la producción de biocombustibles (biodiesel) que existen en los cuerpos de agua marina es que poseen una apreciable cantidad de lípidos (en base seca): Chlorella minutissima (57\%), Chlorella vulgaris (57\%), Nannochloris sp. (40\%), Nannochloropsis oculata (8\%-54\%), Nannochloropsis sp. $(24 \%-60 \%)$ y Tetraselmis suecica $(20 \%-54 \%)$.

En Perú, los residuos pesqueros se producen en grandes cantidades como consecuencia de la intensa actividad de la industria pesquera, que es una de las actividades extractivas importantes del país. Los residuos como vísceras, cabezas, piel, huesos y colas, se cuantifican alrededor del $50 \%$ de la materia prima, resultando finalmente en contaminantes al ser vertidos indiscriminadamente al medio ambiente, provocando problemas de contaminación y enfermedades. Un residuo de importancia es la "sanguaza", efluente constituido por agua, sangre y sólidos de pescado, generado durante su almacenamiento en pozas y transporte hacia los cocinadores en las fábricas de pescado. Sin embargo, estos residuos pueden ser aprovechados eficientemente si reciben un buen tratamiento para transformarlos (Gonzales y Marín, 2005).

En nuestro medio existen industrias que generan fuentes nitrogenadas residuales producto de su actividad, especialmente la industria de la harina de pescado, la cual produce líquidos residuales tales como: "agua de cola", "agua de bombeo" y "sanguaza". La utilización de la sanguaza constituye una alternativa de tecnología limpia de los ambientes pesqueros, pues una tonelada métrica de pescado procesado produce 50 litros de sanguaza y a nivel nacional se han generado más de 500 millones de litros, representando un verdadero problema en las costas del Perú. Por lo tanto, su uso como medio de producción de biomasa algal evitaría la contaminación de puertos. La "sanguaza" contiene cantidades apreciables de proteínas $(6,3 \%)$, grasas $(3,2 \%)$, sólidos solubles e insolubles $(3,5 \%)$, sales minerales $(1,8 \%)$ y otros, las cuales podrían ser aprovechadas para la producción de la biomasa algal (Blas et al., 2011).

EI Perú como país que se encuentra en la orilla del Océano Pacífico y además poseedor de una fuerte industria pesquera que elimina un buen volumen de "sanguaza", esta se podría utilizar en un futuro para la propagación de biomasa algal oleaginosa para la producción 
de biodiesel (biocarburante), además se podrían obtener otros subproductos como la glicerina y producir otros productos a partir de la biomasa algal como alcohol y proteína unicelular. En el Perú se vienen realizando trabajos de investigación en la producción de biodiesel a partir de microalgas en institutos de investigación como IMARPE y en universidades como Universidad Nacional de Trujillo, Universidad Nacional del Santa y otras. A nivel mundial países como Chile, Ecuador, Colombia, Brasil, México, Costa Rica, Estados Unidos de Norteamérica, España y otros, ya tienen implementados plantas pilotos e industrias de producción de biodiesel.

El objetivo del trabajo de investigación fue determinar qué factores biológicos y fisicoquímicos influyen en el aislamiento de microalgas clorofitas de la bahía de Huacho.

\section{MATERIAL Y MÉTODOS}

La población estuvo conformada por flora marina (fitoplancton donde se encuentran las microalgas clorofitas oleaginosas) que están en las aguas marinas de la bahía de Huacho. Se tomaron muestras de agua marina en tres puntos de muestreo o estaciones oceanográficas de la bahía de Huacho con ayuda de un bote con motor el día 03 de Diciembre del 2015. Los tres puntos de muestreo se obtuvieron las coordenadas con un GPS Garmin; estando el primer punto de muestreo o estación oceanográfica ubicado aproximadamente a $850 \mathrm{~m}$ del muelle ENAPU, el segundo a $500 \mathrm{~m}$ del primero y el tercero a $500 \mathrm{~m}$ del segundo con dirección hacia el norte; donde las microalgas que forman parte del fitoplancton fueron filtradas por arrastre impulsado en bote con motor de $40 \mathrm{HP}$ a velocidad de 5 nudos por periodos de 10 minutos en los 3 puntos de muestreo, mediante una red de fitoplancton de 65 a 86 micras. Las muestras fueron colectadas en recipientes de plástico de $100 \mathrm{ml}$ rotulados y conservados en el recipiente cooler en refrigeración para reducir el crecimiento bacteriano, hasta llegar al laboratorio de Biotecnología de la Escuela Profesional de Biología con mención en Biotecnología donde se realizó el trabajo de aislamiento, purificación e identificación de las microalgas clorofitas. Se determinó en el laboratorio el análisis fisicoquímico del agua de mar.
Se aplicaron métodos para una colecta natural de microalgas clorofitas para producir cultivos monoespecíficos o axénicos. Aunque tales métodos son sencillos, sin embargo, la preparación de cultivos axénicos requirió paciencia y perseverancia. Los medios de cultivo enriquecidos usados para fines de aislamiento, purificación y conservación fueron los siguientes: Medio Guillard F/2 (Guillard y Ryther 1963) y el medio Abonofol, ya sea en medios líquidos y sólidos (con $15 \mathrm{~g} / \mathrm{l}$ de agar-agar) a $\mathrm{pH} \mathrm{7,6} \mathrm{y} \mathrm{temperatura}$ ambiental de laboratorio entre $23^{\circ} \mathrm{C}$ a $28,5^{\circ} \mathrm{C}$.

Entre los métodos de aislamiento y purificación que se utilizaron fueron los siguientes: 1) rayado repetidos en placas de agar con solución nutritiva para microalgas: se transfirieron pequeñas gotas de fitoplancton con un asa de siembra, extendiendo por estrías (rompiendo un poco el agar); 2) diluciones sucesivas hasta $10^{-3}$ y subcultivos repetidos en medios sólidos y líquidos. Se establecieron un total de tres placas y tres tubos por cada dilución; 4) pipeteo capilar y lavados sucesivos en placa escavada multipocillos y subcultivos repetidos en medios sólidos y líquidos; 5) cultivo en fotobiorreactores con aire, luz artificial y $\mathrm{CO}_{2}$ constantes en medios líquidos de Guillard $\mathrm{F} / 2$ y Abonofol $(150 \mathrm{ml})$ y $15 \mathrm{ml}$ de muestra de fitoplancton. Luego se hicieron estudios morfológicos de los cultivos microalgales puros para su identificación, utilizando el microscopio óptico compuesto y guías de identificación de algas marinas.

Una vez establecidas las primeras poblaciones microalgales puras, ya sean en medios líquidos o sólidos fueron repicadas en agares y medios líquidos con medios enriquecidos con la solución nutritiva para microalgas. Se emplearon los medios de cultivo: Medio Guillard F/2 (Guillard y Ryther, 1963), dándole las condiciones favorables para el crecimiento de las microalgas clorofitas.

\section{RESULTADOS}

Los tres puntos de muestreo o estaciones oceanográficas en aguas abiertas de la bahía de Huacho, se muestran en la Tabla 1, mientras en la Tabla 2, se muestra el promedio del análisis fisicoquímico de las muestras de agua de mar. 
Tabla 1. Estaciones Oceanográficas en la bahía de Huacho.

\begin{tabular}{ccc}
\hline ESTACIÓN OCEANOGRÁFICA & LATITUD & LONGITUD \\
\hline E1 & $11^{\circ} 07^{\prime} 11.5^{\prime \prime}$ & $77^{\circ} 37^{\prime} 26.6^{\prime \prime}$ \\
E2 & $11^{\circ} 06^{\prime} 56.1^{\prime \prime}$ & $77^{\circ} 37^{\prime} 17.6^{\prime \prime}$ \\
E3 & $11^{\circ} 06^{\prime} 37.8^{\prime \prime}$ & $77^{\circ} 37^{\prime} 19.2^{\prime \prime}$
\end{tabular}

Tabla 2. Análisis fisicoquímico de agua de mar.

\begin{tabular}{cc}
\hline COMPONENTE & CANTIDAD \\
\hline Temperatura & $22^{\circ} \mathrm{C}$ \\
pH & $7,5-7,8$ \\
Salinidad & $35,0 \mathrm{ppm}$ \\
$\mathrm{CO}_{2}$ & $15,0 \mathrm{ppm}$ \\
Amonio & $0,05 \mathrm{ppm}$ \\
Nitrito & $0,05 \mathrm{ppm}$ \\
Nitrato & $0,25 \mathrm{ppm}$ \\
Alcalinidad & $120,0 \mathrm{ppm}$
\end{tabular}

Se trabajaron las tres muestras de fitoplancton muestreados empleando los cuatro métodos de aislamiento e identificación de microalgas clorofitas, presentando resultados negativos con los métodos: rayado repetidos en placas

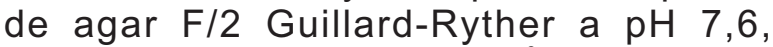
diluciones sucesivas hasta $10^{-3}$ y siembra en agar F/2 Guillard-Ryther a pH 7,6 y pipeteo capilar, lavados sucesivos y siembra en agar F/2 Guillard-Ryther a pH 7,6; o sea no hubo crecimiento de microalgas clorofitas. Mientras que con el cuarto método: siembra en medios líquidos $\mathrm{F} / 2$ Guillard-Rythery Abonofol a $\mathrm{pH}$ 7,6 (150 ml), con aireación, $\mathrm{CO}_{2}$ y luz artificial constante a temperatura ambiente $\left(25{ }^{\circ} \mathrm{C}\right.$ a $28,5^{\circ} \mathrm{C}$ ) por 24 días de incubación utilizando fotobiorreactores de $250 \mathrm{ml}$ de capacidad. Fueron positivos para las muestras de fitoplancton M2 y M3 y negativo para la muestra de fitoplancton M1; los cultivos positivos presentaron las siguientes características: turbidez de color verde de diferentes intensidades, que nos está demostrando presencia de microalgas clorofitas. En la Figura 2 se muestran las condiciones y resultados del crecimiento de las microalgas clorofitas aisladas a partir de muestras de fitoplancton.
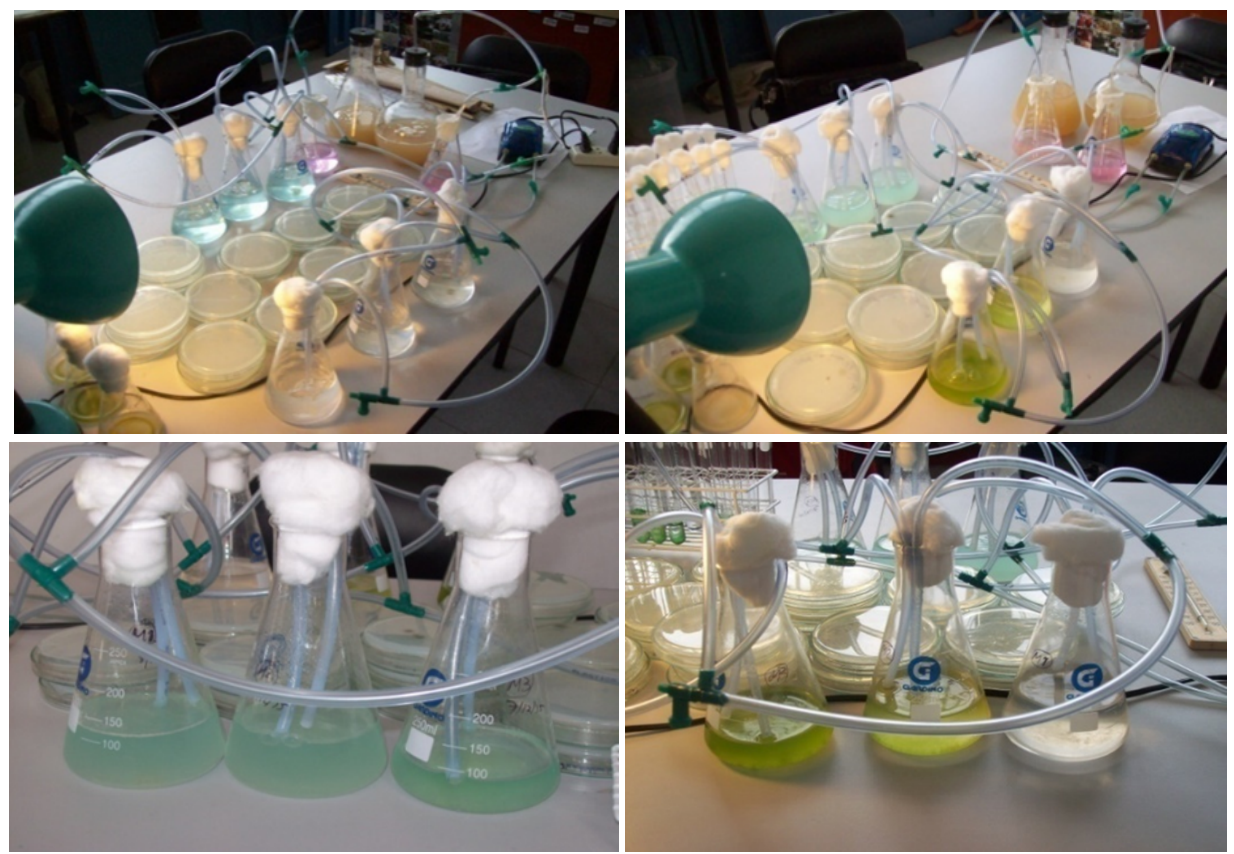

Figura 2. Aislamiento de microalgas clorofitas utilizando fotobiorreactores en medios líquidos (F/2 Guillard-Ryther y Abonofol a pH 7,6) a partir de muestras de fitoplancton. 
Luego los cultivos positivos del método cuarto de 16 días de incubación; fueron resembrados en medios sólidos de F/2 Guillard-Ryther a pH 7,5 por duplicado a temperatura ambiente (26 ${ }^{\circ} \mathrm{C}$ a $28^{\circ} \mathrm{C}$ ) y luz artificial constante por 15 días de incubación, para la purificación de las microalgas clorofitas. Se observó poco crecimiento de microalgaclorofita (colonias de color verde claro) en el medio sólido que provenía del medio líquido Abonofol y de regular a bastante crecimiento de microalgas clorofitas (colonias de color verde claro y verde oscuro) que provenía del medio líquido F/2 Guillard-Ryther. En la Tabla 3 y Figura 3 se presenta el crecimiento de las microalgas clorofitas en medios líquidos y sólidos de F/2 Guillard-Ryther

Tabla 3. Purificación de las microalgas clorofitas aisladas, en medios sólidos de F/2 Guillard- Ryther.

\begin{tabular}{|c|c|c|}
\hline $\begin{array}{l}\text { DÍAS DE } \\
\text { INCUBACIÓN }\end{array}$ & $\begin{array}{l}\text { CULTIVO LIQUIDO } \\
\text { PROVENIENTE }\end{array}$ & $\begin{array}{l}\text { RESULTADO DEL CRECIMIENTO EN } \\
\text { MEDIO SÓLIDO F/2 GUILLARD-RYTHER }\end{array}$ \\
\hline \multirow{4}{*}{8} & M2, Abonofol & Poco crecimiento (+1) \\
\hline & M3, Abonofol & Poco crecimiento $(+1)$ \\
\hline & M2, F/2 Guillrad-Ryther & Crecimiento regular $(+3)$ \\
\hline & M3, F/2 Guillrad-Ryther & Poco crecimiento $(+2)$ \\
\hline \multirow{4}{*}{12} & M2, Abonofol & Poco crecimiento $(+1)$ \\
\hline & M3, Abonofol & Poco crecimiento $(+2)$ \\
\hline & M2, F/2 Guillrad-Ryther & $\begin{array}{l}\text { Buen crecimiento }(+4) \text {. Dos tipos de colonias } \\
\text { (verde claro y verde oscuro) }\end{array}$ \\
\hline & M3, F/2 Guillrad-Ryther & $\begin{array}{l}\text { Crecimiento regular }(+3) \text {. Colonias de color } \\
\text { verde claro. }\end{array}$ \\
\hline \multirow{4}{*}{15} & M2, Abonofol & $\begin{array}{l}\text { Poco crecimiento (+1). Colonias de color } \\
\text { verde claro. }\end{array}$ \\
\hline & M3, Abonofol & $\begin{array}{l}\text { Poco crecimiento (+2). Colonias de color } \\
\text { verde claro. }\end{array}$ \\
\hline & M2, F/2 Guillrad-Ryther & $\begin{array}{l}\text { Buen crecimiento }(+5) \text {. Dos tipos de colonias } \\
\text { (verde claro y verde oscuro). }\end{array}$ \\
\hline & M3, F/2 Guillrad-Ryther & $\begin{array}{l}\text { Crecimiento regular }(+3) \text {. Dos tipos de } \\
\text { colonias (verde claro y verde oscuro). }\end{array}$ \\
\hline
\end{tabular}

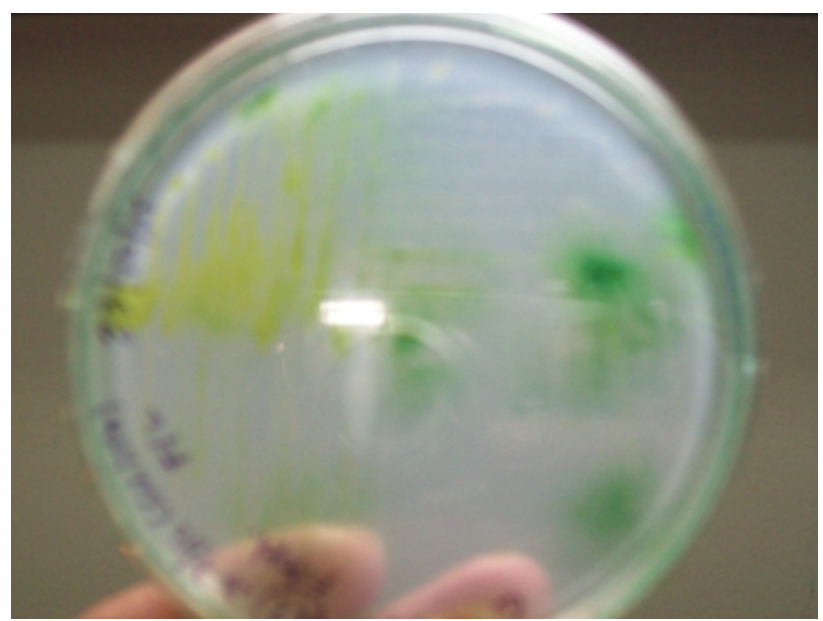

Figura 3. Crecimiento de las microalgas clorofitas en medios sólidos de F/2 Guillard-Ryther a 15 días de incubación. 
De los medios sólidos de F/2 Guillard-Ryther a $\mathrm{pH} 7,5$ sembrados, para la purificación de microalgas clorofitas; después de 15 días de incubación se utilizaron para la identificación de las microalgas clorofitas a través de preparados húmedos observados al microscopio de campo claro. Se identificó dos tipos de colonias de microalgas clorofitas: colonias de color verde claro (Nannochloropsis sp), colonias de color verde oscuro (Chlorella sp., Navicula sp y Spirulina $s p)$. Las microalgas clorofitas fueron conservadas en el medio sólido F/2 GuillardRyther. En la Tabla 4 y Figura 4 se presenta los resultados de identificación de las microalgas clorofitas aisladas.

Tabla 4. Identificación de las microalgas clorofitas aisladas.

\begin{tabular}{cccc}
\hline $\begin{array}{c}\text { Cultivo liquido } \\
\text { proveniente }\end{array}$ & $\begin{array}{c}\text { Numeración del } \\
\text { medio sólido F/2 } \\
\text { Guillard-Ryther }\end{array}$ & $\begin{array}{c}\text { Color de la } \\
\text { colonia de } \\
\text { microalga }\end{array}$ & $\begin{array}{c}\text { Microalga clorofitas } \\
\text { identificada }\end{array}$ \\
\hline $\begin{array}{c}\text { M2, F/2 Guillard- } \\
\text { Ryther }\end{array}$ & 1 & Verde claro & Nannochloropsis sp. \\
\hline M3, F/2 Guillard- & 3 & Verde claro & Nannochloropsis sp. \\
Ryther & 2 & Verde oscuro & Chlorella sp., Navicula sp. \\
Chorella sp., Spirulina sp. \\
\hline
\end{tabular}
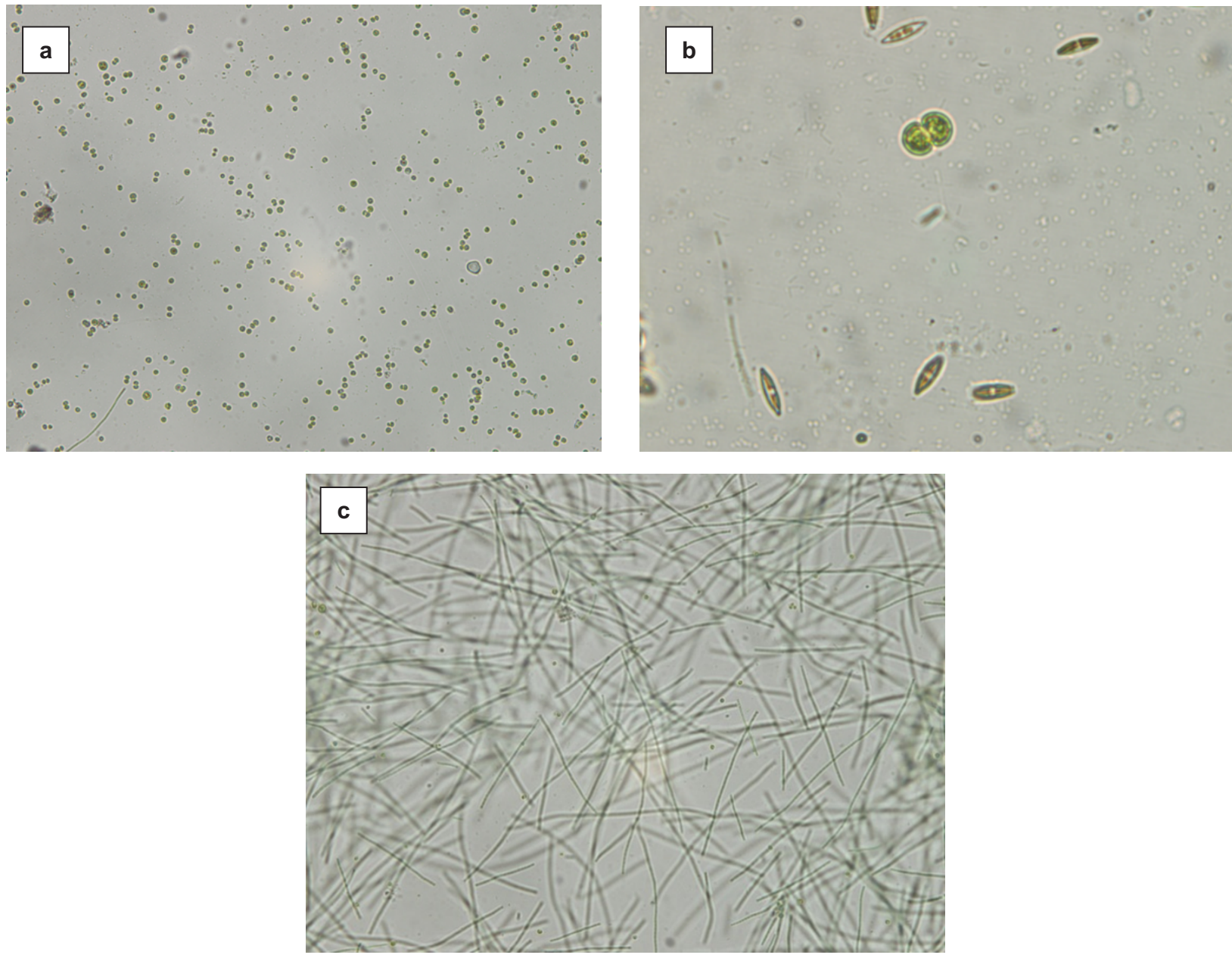

Figura 4. Microalgas clorofitas aisladas en: (a) Nannochloropsis sp, (b) Chlorella sp y Navicula sp y (c) Chlorella sp. y Spirulina sp. 


\section{DISCUSIÓN}

Según Álvarez (1994) y Barajas et al. (2011) señalaron que entre los métodos más usados para el aislamiento de microalgas clorofitas a partir de muestras de fitoplancton concentradas se encuentran: rallado en agar, diluciones seriadas y pipeteo capilar; los cuales no resultaron efectivas para nuestras muestras de fitoplancton porque contuvieron poca cantidad de microalgas clorofitas como se reportó en el estudio. Esto también es señalado por Simon et al. (2009) que menciona las diatomeas (Bacillariophyta), los dinoflagelados (Dinoflagellata), las Haptophyta y las algas verdes (Chlorophyta) podrían contar aproximadamente por el 40 , 40,10 y $6 \%$ de las especies de eucariotas presentes en el fitoplancton marino actual, mientras que la suma de otros grupos contribuiría un $2 \%$.

En este estudio se concluye que las muestras se fitoplancton marino presentaron poca cantidad de microalgas clorofitas (algas verdes) y asimismo se podría utilizar el cultivo en fotobiorreactores en medios líquidos para el aislamiento de microalgas clorofitas para su posterior purificación e identificación.

\section{AGRADECIMIENTOS}

A todas aquellas personas que hicieron posible este estudio, de manera especial a los profesionales colaboradores: Lic. Moraima Y. Romero C., Ing. Luis A. Huayna P. por sus valiosos aportes en cada una de sus especialidades; así como también a los profesionales de IMARPE-Callao en especial a la M.Sc Liz T. García Blázquez por su apoyo técnico y al Ing. Héctor Romero Camarena, Jefe del laboratorio Larval de Camarones de la Facultad de Ingeniería Pesquera de la UNJFSC por el obsequio de cepas de microalgas clorofitas de colección.

\section{REFERENCIAS BIBLIOGRÁFICAS}

Álvarez, M., \& Zarco, J. (1989). Lipids in Microalgae. A review I. Biochemistry. Grasas y Aceites 40(2): 118-145.
Arias, M., Martínez, A. \& Cañizares, R. (2013). Producción de biodiesel a partir de microalgas: parámetros del cultivo que afectan la producción de lípidos. Acta Biol. Colomb., 18(1), 43-68; recuperado el 26 de Setiembre del 2014 , d e www.redalyc.org/pdf/3190/3190280100 04.pdf

Barajas, A., Garzón, L., González, A., Guzmán, A., Kafarov, V., Moreno, N., Nuñez, M., Plata, V. \& Velásquez, G. (2011). Bioprospección de microalgas colombianas para la producción de biodiesel. Recuperado el 26 de Setie mbre del 2014 , de 207.239.251.110:8080/jspui/bitstream/ 1138/3836/1/(MicrosoftWordBIOPROSPECCI.pdf.

Blas, W., Rodríguez, J., Huamán, J., Vigo, W., Moya, W., Sánchez, L. \& Pita, A. (2011). Producción de biomasa de Bacillus thuringiensis $\mathrm{H}-14$ var. israelensis mutante en un medio fermentativo a base de sanguaza. REBIOL., 31(2), 8 págs.

Chisti Y. (2007). Biodiesel form Microalgae. Biotech Adv. 25: 294-306.

Garibay, H. A., Vázquez-Duhalt, R. \& Sánchez, M. P. (2009). Biodiesel a partir de microalgas. Instituto de Biotecnología. Universidad Nacional Autónoma de México. Cuernavaca, Morelos. BioTecnología, 13(3), 38-61.

González, D. \& Marín, M. (2005). Obtención de ensilados biológicos a partir de los desechos del procesamiento de sardinas. Revista Científica FCV-LUZ 15(6), 560-567.

Guillard \& Ryther (1963). Medio F/2. https://www.researchgate.net/.../F2+

Guschina, I. \& Harwood, J. (2006).Lipids and lipid metabolism in eukaryotic algae. 
Progress in Lipid Research, 45,160186.

Hirata, K., Tsujimoto, Y., Namba, T., Ohta, T., Hirayanagi, N., Miyasaka, H., Zenk, M.H. \& Miyamoto, K. (2001). Strong induction of phytochelatin synthesis by zinc in marine green algae. Dunaliella tertiolecta. Journal of Bioscience and Bioengineering 92, 24-29.

Kalia V.C. \& Purohit H.J. (2008). Microbial diversity and genomics in aid of bioenergy. J. Ind. Microbiol. Biotech. $35,403-419$.

Knothe, G., Dunn, R. \& Bagby, M. (1997). Biodiesel: the use of vegetable oils and their derivatives as alternative diesel fuels. ACS Symp Ser. 666, 172-208.

Lee, Y.K. (1997). Commercial production of microalgae en the Asia-Pacific rim. Journal of Applied Phycology 9, 403411.

Ma F.R. \& Hanna M.A., (1999). Biodiesel production: a review. Bioresour Technology 70, 1-15.
Meng X., Yang J., Xu X., Zhang L., Nie Q. \& Xian M. (2008). Biodiesel production from oleaginous microorganisms.Rev. Ener. 34, 1-5.

Simon, N.A.L. Cras; Foulon E. \& Lemée R. (2009). Diversity and evolution of marine phytoplankton. C.R. Biologies 332, 159-170.

Song D., Fu J. \& Shi D. (2008). Exploitation of Oil-Bearing Microalgae for Biodiesel. Chin. Jour. Biotech. 24, 341-348.

Van Gerpen J. (2005). Biodiesel processing and production. Fuel. Process. Technol. 86,1097-1107.

Veziroglu, T.N. \& Barbir F. (1992). Hydrogen: the wonder fuel. Int. J. Hydrogen Energy. 17,391-404.

\section{Correo electrónico:}

lahuayna6255@hotmail.com

Revisión de pares:

Recibido: 03-08-2018

Aprobado: 20-12-2018 